\title{
ARROW-B Type Polarization Splitter with Asymmetric Y-Branch Fabricated by a Self-Alignment Process
}

\author{
Tomokazu Hayakawa, Shuichiro Asakawa, \\ and Yasuo Kokubun, Member, IEEE
}

\begin{abstract}
We have proposed and demonstrated a polarization splitter based on an ARROW-B type waveguide. This device has an asymmetric Y-branch configuration, connecting a waveguide with a large modal birefringence to a low-birefringent waveguide which is channeled by the stripe lateral confinement structure. The device can achieve a high extinction ratio without precise mask alignment, since these two waveguides are connected by self-alignment process. In addition, a low insertion loss is achieved because of adiabatic Y-branching. We designed this device at the wavelength of $1.55 \mu \mathrm{m}$ and fabricated using an RF sputtering. As the result, high extinction ratios of $\mathbf{- 1 9 . 4} \mathrm{dB}$ for TE mode and $-19.0 \mathrm{~dB}$ for TM mode were achieved.
\end{abstract}

Index Terms - ARROW, modal birefringence, optical waveguide, polarization splitter, stripe lateral confinement.

\section{INTRODUCTION}

W AVEGUIDE-TYPE polarizers and polarization splitters are one of essential components in integrated photonics. These can be widely used in integrated-type pickups of magneto-optical disk memories, in polarization independent optical isolators and circulators, and in polarization diversity receivers for coherent transmission systems. One of interesting applications is the polarization division multiplexing in which TE and TM modes carry different optical signals and the polarization splitters are used as multi/demultiplexers of orthogonal polarizations.

There have been reported many polarization splitters for the integrated photonics. These are classified into three types, i.e., the devices based on $\mathrm{LiNbO}_{3}$ [1]-[6], semiconductors [7]-[9], and glass waveguides [10]-[20] from the view point of material. The operational principle of the devices made of $\mathrm{LiNbO}_{3}$ is based on the material birefringence of the crystal. For semiconductors, a large index difference between the semiconductor core and the low index dielectric cladding is utilized. For glass waveguide devices, a large index difference resulting from an additional very high index layer and a selective absorption by metal cladding layer are utilized. In particular the interface with large index difference and the

Manuscript received November 4, 1996; revised March 24, 1997.

T. Hayakawa and Y. Kokubun are with the Department of Electrical and Computer Engineering, Yokohama National University, Yokohama 240, Japan. They are also with Kanagawa Academy of Science and Technology, Kawasaki 213, Japan.

S. Asakawa was with the Yokohama National University. He is now with NTT Opto-electronics Laboratories, Ibaraki-ken 319-11, Japan.

Publisher Item Identifier S 0733-8724(97)05166-9. interface between the metal layer and the dielectric material can achieve a large difference of the boundary condition of the modal field between TE and TM polarizations. Another view point of the classification is the device configuration, and most of glass waveguide devices are realized by the configurations of asymmetric Y-branch [10]-[14] and asymmetric directional coupler [15], [16]. Another type of device configuration is the symmetric directional coupler configuration which utilizes the difference of coupling length for TE and TM modes [17], [18]. In the symmetric directional coupler configuration, high extinction ratio can not be obtained without a precise control of the structure, because the coupling length is sensitive to the cross-sectional structure. In the devices based on the Mach-Zender interferometer (MZI) [19], [20], the phase difference between two arms is adjusted to be $\pi / 2$ for one of TE and TM modes and $-\pi / 2$ for the other mode. This type of device requires a precise trimming of the structure after the fabrication.

The polarization splitters based on the asymmetric directional coupler also requires a precise control of the structure to equate the equivalent indexes of two waveguides for one of the polarizations to achieve the selective coupling. The fabrication error deteriorates the device performance resulting from the decrease of maximum transmittance of coupled power as well as the change of coupling length. In contrast to these, the polarization splitters with the asymmetric Y-branching configuration does not require the precise control of the structure (mainly thickness). This is because the equivalent indexes of two waveguides are required to satisfy only the opposite inequality relation for two polarization modes. These devices employ the adiabatic mode evolution for the coupling between the waveguides, which is called the adiabatic coupling. In the adiabatic coupling, good coupling characteristics can be achieved as long as the cross-sectional structure changes gradually along the propagation direction.

Shani et al. reported a three-dimensional (3-D) waveguide type polarization splitter with the asymmetric Y-branching configuration using dielectric materials [10], [11]. In this device, a channeled waveguide with a thin and very high index core, which has a large modal birefringence, is connected to a channeled waveguide with small birefringence. In the connecting area of the waveguides, a very sharp lateral taper of the core of highly birefringent waveguide is overlapped on the top or the bottom surface of low birefringent waveguide. 


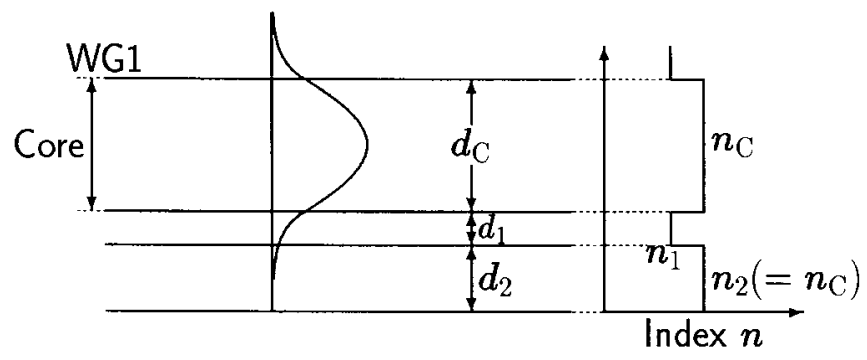

(a)

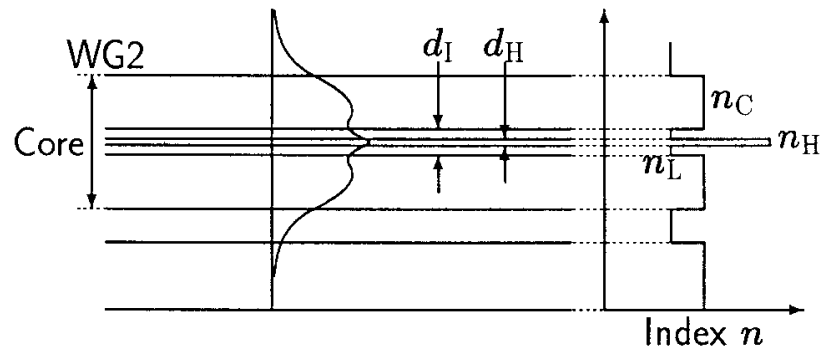

(b)

Fig. 1. Structure of ARROW-B and highly birefringent waveguide. (a) ARROW-B and (b) ARROW-B with multi-intermediate layer.

In the overlap area, the mode of highly birefringent waveguide spreads out widely due to the tapered width of the core, and the mode can be coupled to the low birefringent waveguide. However, since the field amplitude at the bottom or the top of WG1 at which the core of WG2 is located, is not so large in the overlap area, the interaction between the modes in WG1 and WG2 is not so strong. This results in a long device size required for the adiabatic evolution of the mode. In addition, this device requires a very fine lithography technique to get a sharp tip point of tapered area of highly birefringent waveguide.

To overcome the above difficulties, the authors propose a new polarization splitter made of a glass waveguide.

\section{PRINCIPLE}

Fig. 1 shows the comparison of a conventional lowbirefringent single-mode waveguide and a high birefringent waveguide with multilayer core. The waveguide $\sharp 1$ (WG1 for short) shown in Fig. 1(a) is a normal ARROW-B [21]. In the waveguide $\sharp 2$ (WG2 for short) shown in Fig. 1(b), a multilayer which consists of a high index layer sandwiched by low index layers is inserted at the center of the core layer. We call this multilayer the multi-intermediate layer. The equivalent index of the WG1 is designated as $n_{\mathrm{eq} 1}$ for both TE and TM polarizations, because the birefringence of WG1 is small. On the other hand, the equivalent index of $\mathrm{TE}_{0}$ mode $n_{\mathrm{eq} 2}^{(\mathrm{TE})}$ is larger than that of $\mathrm{TM}_{0}$ mode $n_{\mathrm{eq} 2}^{(\mathrm{TM})}$ in the WG2, due to the so-called modal birefringence. Since this birefringence can be controlled by the thickness of high index layer as will be described in the following section, we can arrange the equivalent indexes so as to satisfy the following inequality relation:

$$
n_{\mathrm{eq} 2}^{(\mathrm{TM})}<n_{\mathrm{eq} 1}<n_{\mathrm{eq} 2}^{(\mathrm{TE})} .
$$

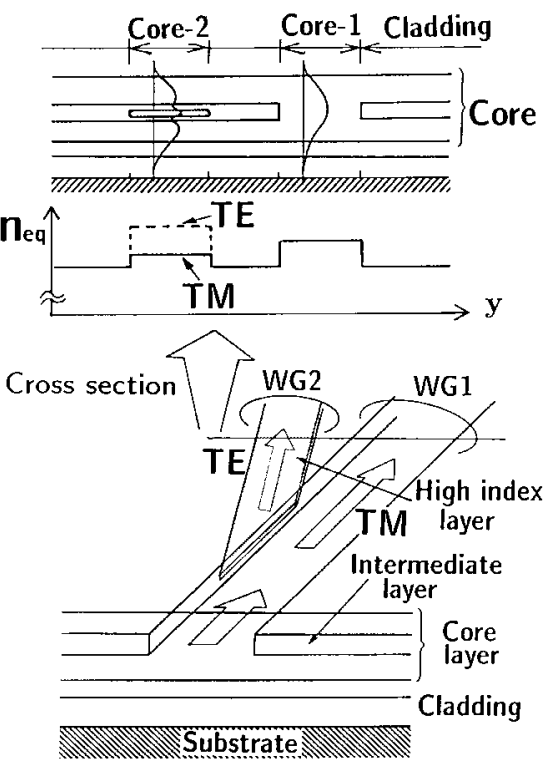

Fig. 2. Structure of polarization splitter and schematic equivarent index profile.

Generally speaking, the light is guided to a waveguide with higher equivalent index in the Y-branching waveguide [22]. Therefore, when the WG2 is connected to the WG1 so as to form an asymmetric Y-branch, TE mode is guided toward the WG2 and TM mode is guided to the WG1 without coupling to the WG2. The polarization splitters utilizing the asymmetric directional coupler [19], [20] have a problem that the precise control of structure is required to adjust the equivalent indexes of two waveguides to achieve the selective coupling. In contrast to this, the polarization splitters with the Y-branching configuration including our device do not require such precise control of the structure (mainly thickness) because these can operate as long as the equivalent indexes of waveguides satisfy the relation (1).

\section{STRUCTURE}

The structure of our new polarization splitter is shown in Fig. 2. The upper side of this figure illustrates the crosssectional structure in the transverse direction of the waveguides. The WG1 is the 3-D ARROW-B with the stripe lateral confinement (SLC) structure [23], [24]. Another WG2 is the ARROW-B which is channeled by inserting the strip of a very thin and very high index layer in the middle of the low index intermediate layer in the cladding region of WG1. The high index layer does not contribute to confine the field in the vertical direction, because this high index layer is very thin. The polarization dependence of the equivalent indexes in the Core- 1 region $n_{\text {eq1 }}$ and the cladding region $n_{\text {eq }}^{\text {(clad) }}$ are negligibly small. On the other hand, the equivalent index in the Core-2 region $n_{\mathrm{eq} 2}$ significantly splits into $n_{\mathrm{eq}}^{(\mathrm{TE})}$ and $n_{\mathrm{eq}}^{(\mathrm{TM})}$ for TE and TM modes due to the existence of the multi-intermediate layer [24]. Therefore, WG2 acts as the birefringent waveguide. The schematic equivalent index profiles is shown in the middle of Fig. 2. When the equivalent index of the WG2 is higher than that of the WG1 for TE mode 


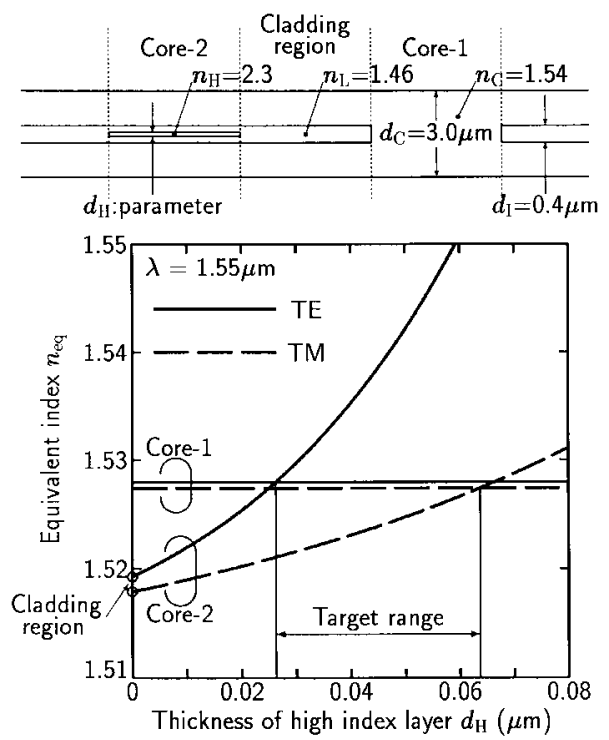

Fig. 3. Caluculated results of equivalent indexes in the Core-1 and the Core- 2 regions at $\lambda=1.55 \mu \mathrm{m}$.

and opposite for TM mode, this device acts as a polarization splitter.

In this polarization splitter, the scattering loss of WG2 having the high index layer can be reduced because this layer plays the role of the origin of large modal birefringence but does not confine the field into this layer. The key point to reduce the scattering loss is the introduction of the outer core layer which mainly confines the modal field. Although the very sharp tip point of the tapered core is required for the adiabatic branching, it is formed automatically by the etching of WG1. The core of WG2 completely contacts with the side of the core of WG1 with self-alignment. This results in the lack of precise mask alignment in the lithography process. Furthermore, because WG1 and WG2 have similar field profiles owing to the same thicknesses of the cores, the device length can be shorter than Shani's device due to the strong interaction of the modes.

\section{ANALYSIS AND DESIGN}

\section{A. Equivalent Index}

Fig. 3 shows the calculated results of the equivalent indexes in the Core- 1 and the Core- 2 regions at the wavelength $\lambda=1.55 \mu \mathrm{m}$. The refractive indexes were assumed to be 1.54 of NA45 glass [25] for the core layer and the second cladding layer, 1.46 of $\mathrm{SiO}_{2}$ for the low index layer and the first cladding layer, and 2.3 of $\mathrm{TiO}_{2}$ for the high index layer. The thicknesses of the intermediate layer and the core were assumed to be 0.4 and $3 \mu \mathrm{m}$, respectively. The thicknesses of the first cladding and the second cladding were 2.6 and $1.5 \mu \mathrm{m}$, respectively, to ensure the radiation loss of ARROW-B to be less than $0.1 \mathrm{~dB} / \mathrm{cm}$. The total thickness of low index layer in the multi-intermediate layer was assumed to be a constant value of $0.4 \mu \mathrm{m}$.

When the thickness of high index layer $d_{\mathrm{H}}$ increases, the equivalent index of Core- 2 becomes higher than that of Core- 1 .

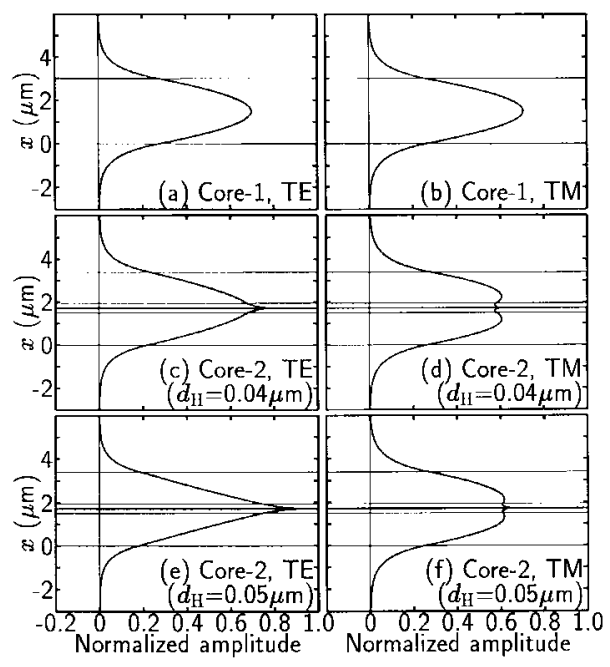

Fig. 4. Field profiles of fundamental mode in the Core-1 and the Core-2 regions.

In addition, the equivalent index of TE mode is much greater than that of TM mode due to the modal birefringence. The equivalent index in the cladding region $n_{\text {eq }}^{\text {(clad) }}$ corresponds to the case of $d_{\mathrm{H}}=0 \mu \mathrm{m}$. The splitter condition (1) can be obtained in the wide range of $d_{\mathrm{H}}=0.027-0.063 \mu \mathrm{m}$. As long as this condition is satisfied, the TM mode passes straight through the Core-1, while TE mode is guided into the Core-2.

\section{B. Field Profiles}

The field profiles of the fundamental mode in the Core1 and the Core-2 regions are shown in Fig. 4(a)-(f). In the Core- 2 region with multi-intermediate layer, the field profiles are quite different depending on the polarization as shown in Fig. 4(c)-(f). The field of TE mode is well confined in the high index layer, while the field of TM mode is forced out from the multi-intermediate layer. Therefore, the equivalent index is large for TE mode, while opposite for TM mode. Comparing the field profiles of TE mode shown in Fig. 4(c) and (e), the field profile in the Core-2 region with $d_{\mathrm{H}}=0.04 \mu \mathrm{m}$ is less deformed by the intermediate layer than the case of $d_{\mathrm{H}}=0.05 \mu \mathrm{m}$ [see Fig. 4 (e)], and is very close to that in the Core-1 region with the monolayer core. On the other hand, when $d_{\mathrm{H}}=0.05 \mu \mathrm{m}$, the field profile is much confined in the high index layer, and so this mode may suffer a large scattering loss. Therefore, it can be concluded that the thickness $d_{\mathrm{H}}$ is desirable to be thinner in the allowed range of $d_{\mathrm{H}}$. In this study, we designed as $d_{\mathrm{H}}=0.04 \mu \mathrm{m}$ so that the difference between $n_{\mathrm{eq} 2}^{(\mathrm{TE})}$ and $n_{\mathrm{eq} 1}$ is almost the same as that between $n_{\mathrm{eq} 1}$ and $n_{\mathrm{eq} 2}^{(\mathrm{TM})}$.

\section{BPM Simulation}

Fig. 5(a) and (b) show the results of the two-dimensional (2D) BPM simulation. The three-dimensional waveguide structure was replaced by the 2-D structure, using the equivalent index method. The branching angle $\theta$ is $0.3^{\circ}$ and the core width is $5 \mu \mathrm{m}$. When the branching angle is $0.3^{\circ}$, the waveguides are separated by $15 \mu \mathrm{m}$ (center to center) at the position $3 \mathrm{~mm}$ 


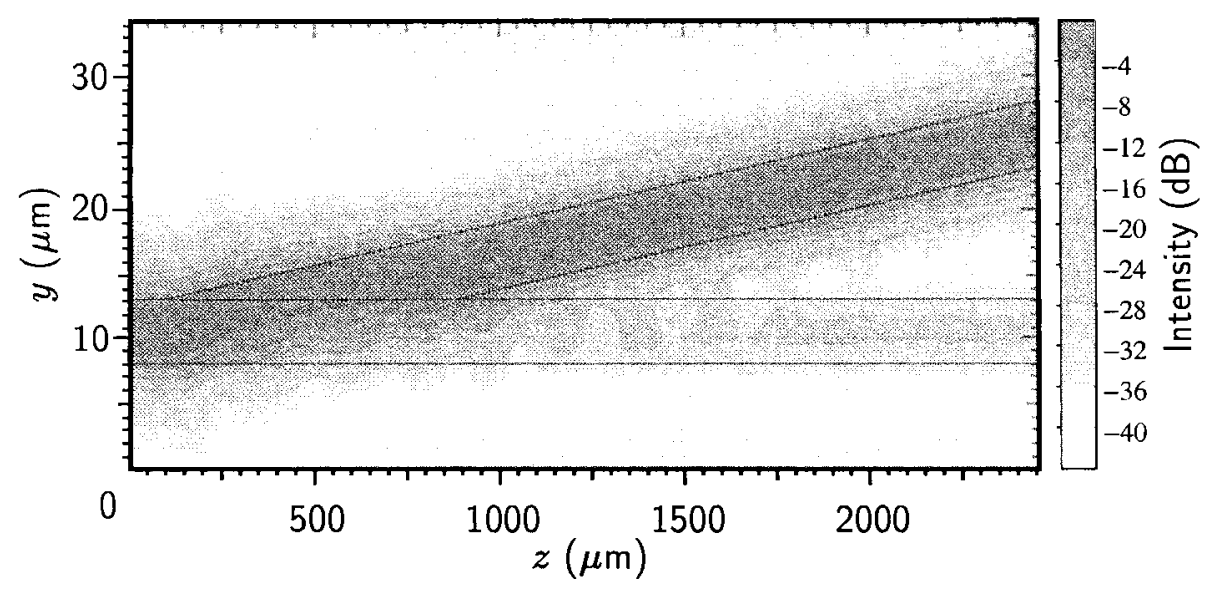

(a)

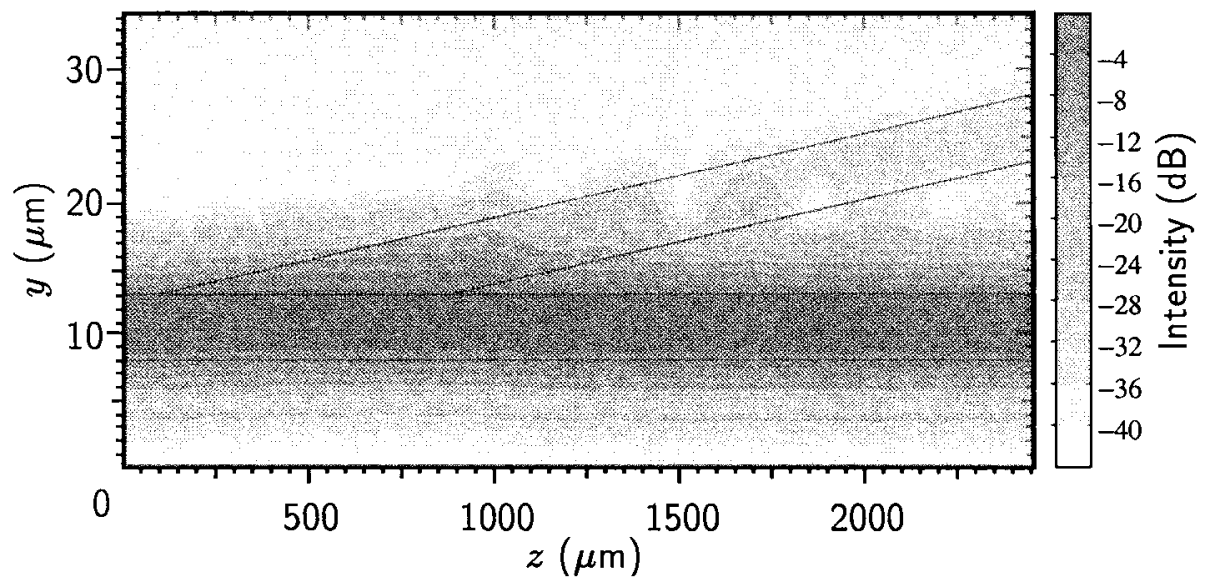

(b)

Fig. 5. Results of two-dimensional BPM simulation. The branching angle was assumed to be $0.3^{\circ}$. (a) TE mode input and (b) TM mode input.

from the starting point of the branching. Now, let us define the extinction ratios for TE and TM modes by

$$
\begin{gathered}
\Gamma_{(\mathrm{TE})}=10 \log \frac{P_{1(\mathrm{TE})}}{P_{2(\mathrm{TE})}} \quad[\mathrm{dB}] \\
\Gamma_{(\mathrm{TM})}=10 \log \frac{P_{2(\mathrm{TM})}}{P_{1(\mathrm{TM})}} \quad[\mathrm{dB}]
\end{gathered}
$$

where $P_{1}$ and $P_{2}$ are the output power from the WG1 and the WG2, respectively. The theoretical extinction ratios were evaluated to be $-27 \mathrm{~dB}$ for TM mode and $-21 \mathrm{~dB}$ for TE mode. When the branching angles were $0.5^{\circ}$ and $1.0^{\circ}$, these ratios were $-18 \mathrm{~dB}$ and $-12 \mathrm{~dB}$ for TE mode, respectively. However, these ratio were almost the same for TM mode in contrast to TE mode. This is because the path for TM mode is straight while that for TE mode includes a bend at the asymmetric branching point.

\section{FABRICATION}

Fig. 6 shows the outline of the fabrication process of the device. All the layers of the device were deposited by an RF sputtering technique. The core and the second cladding material were NA45 glass, the first cladding and the low index layers were $\mathrm{SiO}_{2}$ and the high index layer was $\mathrm{TiO}_{2}$. In the first step of the process, the layers from the second cladding to the low index layer of half of the multi-intermediate layer were deposited on the $\mathrm{Si}$ substrate. Next, the high index $\mathrm{TiO}_{2}$ layer was deposited on the low index $\mathrm{SiO}_{2}$ layer and was patterned by the photolithography technique to form the Core-2 region. Then the upper $\mathrm{SiO}_{2}$ layer was deposited as shown in Fig. 6(a).

Next, the whole intermediate layer was etched down to the lower half core to form the Core-1 region by using the reactive ion etching (RIE) technique with $\mathrm{CF}_{4}$ gas. In the RIE process, chromium was used as the mask. Since the etching rate of NA45 glass is one tenth smaller than that of $\mathrm{SiO}_{2}$, the etching can be stopped almost at the top surface of the lower half of core layer. In this process, the high index $\mathrm{TiO}_{2}$ layer in the region where the Core- 1 and Core- 2 regions are overlapped was also eliminated as shown in Fig. 6(b). Therefore, the branching region can be formed smooth enough automatically and the edge of the branching waveguide is sharp enough. This very sharp branching of the Core- 2 region results in the reduction of excess loss. In addition, the Core- 2 can be completely connected to the side of the Core- 1 with selfalignment. This eliminates the precise mask alignment in the lithography process.

Last, the upper half of the core layer was deposited to bury the intermediate layer, and the $\mathrm{SiO}_{2}$ cover cladding of $1.0 \mu \mathrm{m}$ thick was formed on the top of the core layer as 


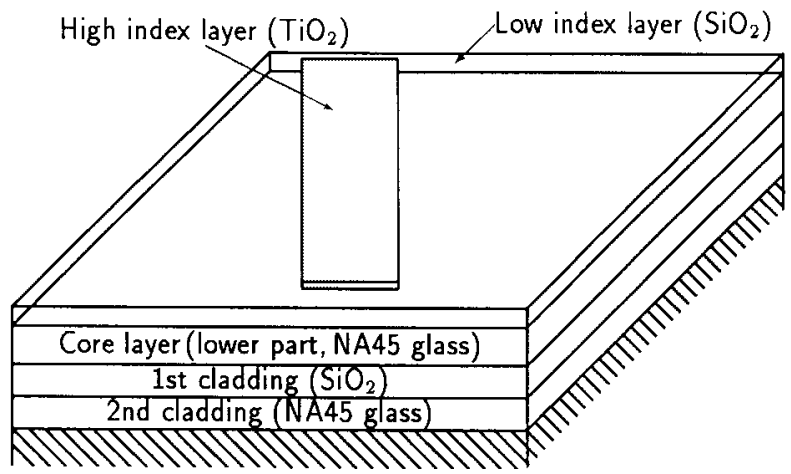

(a)

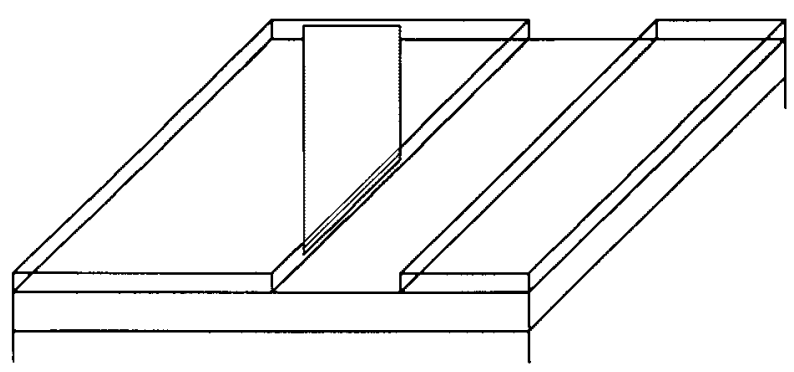

(b)

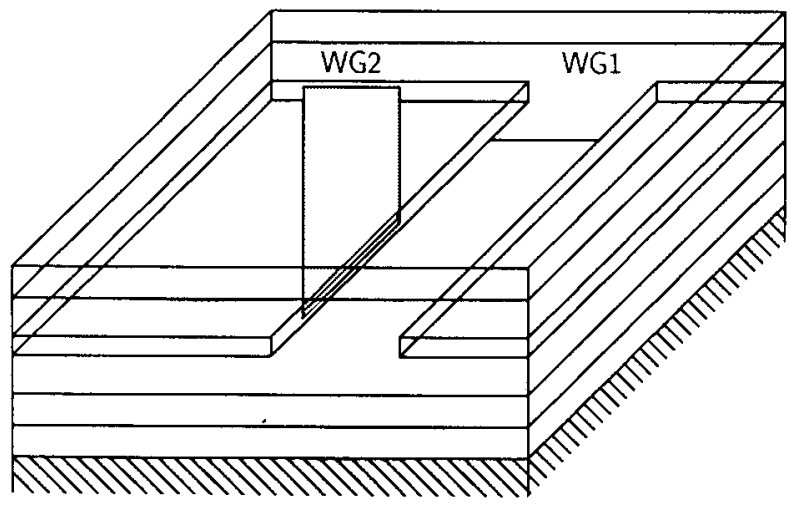

(c)

Fig. 6. Outline of the fabrication process: (a) deposition of lower half layers of the device and the patterning of high index layer to form the Core-2 region, (b) reactive ion etching of the intermediate layer, and (c) deposition of the upper core layer and the upper cladding layer.

shown Fig. 6(c). The core width and thickness were 5 and $3 \mu \mathrm{m}$, respectively. Other parameters were $d_{\mathrm{I}}=0.4 \mu \mathrm{m}$, $d_{\mathrm{H}}=0.04 \mu \mathrm{m}, d_{1}=2.6 \mu \mathrm{m}, d_{2}=1.5 \mu \mathrm{m}$ and $\theta=0.3^{\circ}$, and these values are the same as those assumed in the design and the BPM simulation. The device length was $3 \mathrm{~mm}$ when the waveguides were separated by $15 \mu \mathrm{m}$ (center to center). This value is almost the same length as Shani's device. This is because the branching consists of straight waveguides, and the length will be reduced by bending waveguides.

In this experiment, we adopted the core thickness of $3 \mu \mathrm{m}$. However, it is also easy to design the device with a thick core.

\section{MEASURED RESUlTS}

The performance of the fabricated devices was measured at the wavelength of $1.55 \mu \mathrm{m}$. The light of about $45^{\circ}$ linear polarization was incident on the input end from a fiber. The

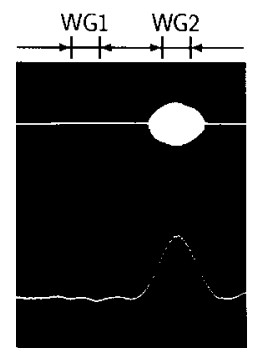

(a)

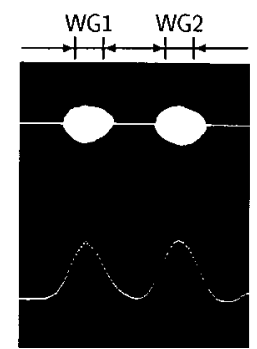

(b)

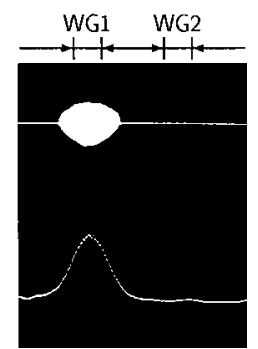

(c)
Fig. 7. Near field patterns of the output light. (a) TE mode input, (b) TE + TM modes input, and (c) TM mode input.

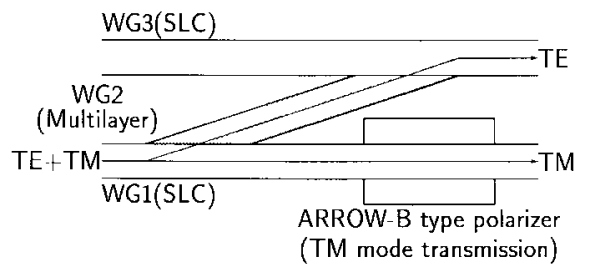

Fig. 8. Improved structure of the polarization splitter with high performance.

output light was collimated by a lens and the extinction ratio was measured using a polarizer. The near field patterns of the output light from the end of the waveguides are shown in Figs. 7(a)-(c). The measured extinction ratios were $-19.4 \mathrm{~dB}$ for TE mode and $-19.0 \mathrm{~dB}$ for TM mode. The insertion losses were $1.1 \mathrm{~dB}$ for TE mode and $0.6 \mathrm{~dB}$ for TM mode, which were measured by the cut back method. The insertion loss of TE mode was larger than that of TM mode because of the scattering loss at the multi-intermediate layer. Although these results were not as good as Shani's results, the improvement of performance is possible by adopting an improved structure as shown in Fig. 8 and also by the reduction of scattering loss.

\section{CONCLUSION}

We proposed and demonstrated a polarization splitter with an asymmetric Y-branching configuration. In this device, a 3-D ARROW-B with the SLC structure and a channeled birefringent waveguide are connected in the lateral direction to form an asymmetric Y-branch. The polarization splitting function is achieved by the selective branching from the ARROW-B to the multilayer waveguide due to the large birefringence of the multilayer waveguide. The measured extinction ratios were $-19.4 \mathrm{~dB}$ and $-19.0 \mathrm{~dB}$ for TE and TM modes, respectively, at the wavelength of $1.55 \mu \mathrm{m}$. Since the splitting condition is satisfied in a wide tolerance of structure, the precise control of the structure is not required to achieve a high performance. The measured insertion losses were 1.1 and $0.6 \mathrm{~dB}$ for TE and TM modes, respectively.

This device can be easily extended to a polarization splitter with higher performance as shown in Fig. 8. The TE mode in the WG1 is coupled to the WG3 through the WG2. The extinction ratio for TE mode is improved by twice in decibel compared with the original configuration owing to the fact that TE mode experiences the selective branching twice in this pass. In addition, the length of the WG2 which causes the scattering loss can be reduced. Since the structure of this 
device has the same structure in the vertical direction with the ARROW-B polarizer [24] the extinction ratio for TM mode can be improved by adding the ARROW-B polarizer to this configuration. The polarizer eliminates the slightly remained TE mode at the output port. These improvements of the extinction ratio allows the substantial increase of the branching angle, and consequently the device length which is defined by the distance between the input and output ports can be reduced.

\section{ACKNOWLEDGMENT}

The authors express their sincere thanks to Dr. M. Maeda of Hitachi Co. Ltd. for supplying distributed feedback laser diode (DFB-LD).

\section{REFERENCES}

[1] N. Goto, and G. L. Yip, "A TE-TM mode splitter in $\mathrm{LiNbO}_{3}$ by proton exchange and Ti diffusion," J. Lightwave Technol., vol. 7, pp. 1567-1574, Oct. 1989

[2] M. Masuda, and G. L. Yip, "An optical TE-TM mode splitter using a $\mathrm{LiNbO}_{3}$ branching waveguide," Appl. Phys. Lett., vol. 37, no. 1, pp. 20-22, 1980.

[3] J. E. Baran, and D. A. Smith, "Adiabatic $2 \times 2$ polarization splitter on $\mathrm{LiNbO}_{3}$, , IEEE Photon. Technol. Lett., vol. 4, no. 1, pp. 39-40, 1992.

[4] H. Nakajima, T. Horimatsu, M. Seino, and I. Sawaki, "Crosstalk charactaristics of $\mathrm{Ti}-\mathrm{LiNbO}_{3}$ intersecting waveguides and their application as TE/TM mode splitters," IEEE J. Quantum Electron., vol. QE-18, pp. 771-776, Apr. 1982.

[5] O. Mikami, "LiNbO 3 coupled-waveguided TE/TM mode splitter," Appl. Phys. Lett., vol. 36, no. 7, pp. 491-492, 1980

[6] K. G. Han, S. Kim, and S. S. Shoi, "Controllable integrated-optic polairzation splitter-combiner," Opt. Lett., vol. 15, no. 2, pp. 108-109, 1990.

[7] J. J. G. M. van der Tol, J. W. Pedersen, E. G. Metaal, Y. S. Oei, F. H. Groen, and I. Moerman, "Mode evolution type polarization splitter on InGaAsP/InP," IEEE Photon. Technol. Lett., vol. 5, pp. 1412-1414, Dec. 1993.

[8] S. Somekh, E. Garmire, A. Yariv, H. L. Garvin, and R. G. Hunsperger, "Channel optical waveguides and directional couplers in GaAs-inbedded and ridged," Appl. Opt., vol. 13, no. 2, pp. 327-330, 1974.

[9] P. Albrecht, M. Hamacher, H. Heidrich, D. Hoffmann, H.-P. Nolting, and C. M. Weinert, "TE/TM mode splitter on InGaAsP/InP," IEEE Photon. Technol. Lett., vol. 2, pp. 114-115, Feb. 1990.

[10] Y. Shani, C. H. Henry, R. C. Kistler, R. F. Kazarinov, and K. J. Orlowsky, "Integrated optic adiabatic polarization splitter on silicon," Appl. Phys. Lett., vol. 56, no. 2, pp. 120-121, 1990.

[11] Y. Shani, C. H. Henry, R. C. Kistler, R. F. Kazarinov, and K. J. Orlowsky, "Integrated optic adiabatic devices on silicon," IEEE J. Quantum Electron., vol. 27, pp. 556-566, Mar. 1991.

[12] K. Matsubara, and H. Yajima, "Y-branch TE-TM mode splitter with multilayered structure," in Third Microoptics Conf. Tech. Dig., Yokohama, Japan, 1991, L13, pp. 202-205.

[13] T. Mizumoto, N. Iwakiri, T. Kaneko, and Y. Naito, "Analytical and experimental study of waveguide optical polarization splitter with langmuir-blodgett cladding layer," J. Lightwave Technol., vol. 10, pp. 1807-1813, Dec. 1992.

[14] R. M. de Ridder, A. F. M. Sander, A. Driessen, and J. H. J. Fluitman, "An integrated optic adiabatic TE/TM mode splitter on silicon," $J$. Lightwave Technol., vol. 11, pp. 1806-1811, Nov. 1993.

[15] M. Kobayasi, H. Terui, and K. Egashiro, "An optical waveguide TE-TM mode splitter," Appl. Phy. Lett., vol. 32, no. 5, pp. 300-302, 1978.

[16] K. Thyagarajam, and S. Pilevar, "Resonant tunneling three-waveguide polarization splitter," J. Lightwave Technol., vol. 10, pp. 1334-1337, Oct. 1992.

[17] M. Eisenmann, and E. Weidel, "Single-mode fused biconical coupler optimized for polarization beamsplitting," J. Lightwave Technol., vol. 9, pp. 853-858, July 1991.

[18] K. Thyagarajam, S. Diggavi, and A. K. Ghatak, "Design and analysis of a novel polarization splitting directional coupler," Electron. Lett., vol. 24 , no. 14 , pp. $869-870,1988$
[19] Y. Shani, C. H. Herry, R. C. Kistler, and K. Orlowsky, "Four-port integrated optic polarization splitter," Appl. Opt., vol. 29, no. 3, pp. 337-339, 1990

[20] M. Okuno, A. Sugita, K. Jinguji, and M. Kawachi, "Birefringence control of silica waveguides on $\mathrm{Si}$ and its application to a polarizationbeam splitter/switch," J. Lightwave Technol., vol. 12, no. 4, pp. 625-633, 1994.

[21] T. Baba and Y. Kokubun, "New polarization-insensitive antiresonant reflecting optical waveguide (ARROW-B)," IEEE Photon. Technol. Lett., vol. 1, pp. 232-234, Aug. 1989.

[22] W. K. Burns and A. F. Milton, "Mode conversion in planar-dielectric separating waveguides," IEEE J. Quantum Electron., vol. QE-11, pp. 32-39, Jan. 1975

[23] T. Baba, Y. Kokubun, and Y. Mera, "A novel 3-dimensional ARROW by thin film patterning-stripe lateral confinement of ARROW," in 10th Topical Meeting Integr. Guided-Wave Opt., Houston, TX, 1989, TuBB-5.

[24] S. Asakawa and Y. Kokubun, "ARROW-B type polarizer utilizing multilayer stripe lateral confinement," IEEE Photon. Technol. Lett., vol. 7, pp. 38-40, Jan. 1995.

[25] NA45 glass is made by HOYA Corp., and has the same composition as C7059 glass.

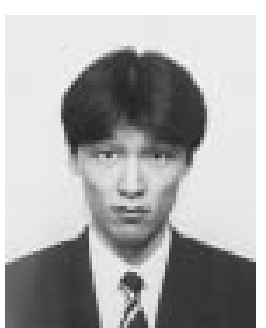

Tomokazu Hayakawa was born in Ibaraki, Japan, on February 5, 1973. He received the B.E. degree in electrical and computer engineering from Yokohama National University, Yokohama, Japan, in 1995. He is currently working toward the M.E. degree at the Yokohama National University.

His main interests include waveguide-type functional devices and optical interconnects.

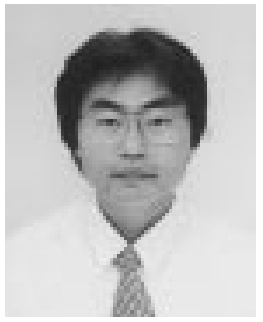

Shuichiro Asakawa was born in Ibaraki, Japan, on August 26, 1966. He received the B.E., M.E., and Dr.Eng. degrees in electrical and computer engineering from Yokohama National University, Yokohama, Japan, in 1990, 1992, and 1995, respectively.

He joined NTT Opto-electronics Laboratories in 1995 , and is working on integrated photonic devices.

Dr. Asakawa is a member of the Japan Society of Applied Physics, the Institute of Electronics, Information and Communication Engineers (IEICE)

of Japan.

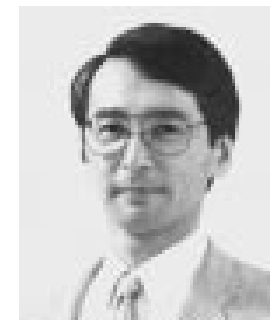

Yasuo Kokubun (M'85) was born in Fukushima, Japan, on July 7, 1952. He received the B.E. degree from Yokohama National University, Yokohama, Japan, in 1975, and the M.E. and Dr.Eng. degrees from Tokyo Institute of Technology, Tokyo, Japan, in 1977 and 1980, respectively.

After he worked for the Research Laboratory of Precision Machinery and Electronics, Tokyo Institute of Technology, as a Research Associate from 1980 to 1983 , he joined the Yokohama National University as an Associate Professor in 1983, and is now a Professor of the Department of Electrical and Computer Engineering. His current research is in integrated photonics, especially waveguide-type functional devices. From 1984 to 1985, he was with AT\&T Bell Laboratories, Holmdel, NJ, as a Visiting Reseacher and was engaged in a novel waveguide on semiconductor substrate (ARROW) for integrated optics.

Dr. Kokubun is a member of the Institute of Electronics, Information and Communication Engineers (IEICE) of Japan, the Japan Society of Applied Physics, and the Optical Society of America. 